\title{
Sharp Superconductor-Insulator Transition in Short Wires
}

\author{
Dganit Meidan, ${ }^{1}$ Yuval Oreg, ${ }^{1}$ and Gil Refael ${ }^{2}$ \\ ${ }^{1}$ Department of Condensed Matter Physics, Weizmann Institute of Science, Rehovot, 76100, Israel \\ ${ }^{2}$ Department of Physics, California Institute of Technology, Pasadena, California 91125, USA
}

(Received 28 November 2006; published 1 May 2007)

\begin{abstract}
Recent experiments on short MoGe nanowires show a sharp superconducting-insulating transition at the universal resistance $R_{Q}=h /\left(4 e^{2}\right)$, contrary to the expectation of a smooth temperature dependence of the resistance for such Josephson-like systems. We present a self-consistent renormalization-group treatment of interacting quantum phase slips in short superconducting wires, which reproduces this sharp universal transition. Our method should also apply to other systems in the sine-Gordon universality class, in the previously inaccessible intermediate-coupling regime.
\end{abstract}

One of the most intriguing problems in low-dimensional superconductivity is the understanding of the mechanism that drives the superconductor-insulator transition (SIT). Experiments conducted on quasi-one-dimensional (1D) systems have shown that varying the resistivity and dimensions of thin metallic wires can suppress superconductivity $[1,2]$, and in certain cases lead to an insulating-like behavior [3-6].

Particularly interesting are recent experiments conducted on short MoGe nanowires [6] that explore the SIT tuned by the wire's normal state resistance with a critical resistance $R_{c} \approx R_{Q}$. The universal critical resistance may suggest that at a temperature much lower than the meanfield transition temperature, $T \ll T_{c}$, the wire acts as a superconducting (SC) weak link resembling a Josephson junction (JJ) connecting two SC leads. Schmid [7] and Chakravarty [8] showed that a JJ undergoes a SIT as a function of the junction's shunt resistance, $R_{s}$, with a critical resistance of $R_{Q}$, and that the resistance across the junction obeys the power law $R(T) \propto T^{2\left[\left(R_{Q} / R_{s}\right)-1\right]}$. The theory was later extended to JJ arrays and SC wires [9-11]. Within these theories, a similar power-law temperature dependence of the resistance at low temperature prevails.

Contrary to this prediction, Bollinger et al. [6] observe that the resistance of quasi-1D MoGe nanowires exhibits a much stronger temperature dependence, even close to the SIT. In fact, the resistance could be fitted with a modified LAMH theory $[12,13]$ of thermally activated phase slips down to very low temperatures. Nevertheless, for these narrow wires it appears that the LAMH theory is valid only in a narrow temperature window [14,15]. Moreover, the LAMH analysis does not explain the appearance of a critical resistance $R_{c} \approx R_{Q}$.

We propose an approach that captures both the critical resistance of $R_{c} \approx R_{Q}$ at the SIT and the sharp decay of the resistance as a function of temperature. As in previous works, we treat the SIT in nanowires as a transition gov- erned by quantum phase-slip (QPS) proliferation. This picture alone, however, cannot account for the observed strong temperature dependence of the resistance. We note that in SC wires, collective excitations involving phase and amplitude fluctuations have considerably different propagation velocities. Hence, there is an intermediate regime for which QPSs that occur in different sections of the wire create phase fluctuation that propagate very fast to the leads, altering the effective resistance that shunt other phase slips. We claim that the key ingredient left out in previous works is the inclusion of interactions between QPSs in such a finite-size wire, especially when the phase-slip population is dense-a situation that occurs in thin wires at $T \lesssim T^{*}$ (see Ref. [11] and Ref. [14] for a definition of $T^{*}$ ).

We treat these interactions in a mean-field-type approximation: when analyzing the behavior of a small segment of the wire, we include in its effective shunt resistance the resistance due to phase slips elsewhere in the wire. This scheme is motivated by numerical analysis of a related problem, an interacting pair of resistively shunted JJs [16]. Primarily, this self-consistent treatment produces the sharp temperature dependence of the resistance. In addition, we include the effects of the Bogoliubov quasiparticles, which couple to the potential gradient created by each phase slip [17]. Consequently, the resistance obtained in the experiment can be fitted without resorting to the LAMH theory beyond its limit of validity. This self-consistent approximation of phase-slip interactions should be applicable to similar multiple sine-Gordon models in the theoretically challenging intermediate-coupling regime.

The microscopic action for a SC wire can be obtained from the BCS Hamiltonian by a Hubbard-Stratanovich transformation followed by an expansion around the saddle point [18]. In the limit of low energy scales, $\omega, D q^{2} \ll \Delta_{0}$, this yields [19,20] 


$$
\begin{aligned}
S= & N_{0} A \Delta_{0}^{2} \int_{0}^{L} d x \int_{0}^{1 / T} d \tau\left\{\frac{\rho^{2}}{2}\left[\ln \left(\rho^{2}\right)-1\right]\right. \\
& \left.+2 \xi_{0}^{2} \rho^{2}\left[\phi^{\prime 2}+\frac{1}{v_{\phi}^{2}} \dot{\phi}^{2}\right]+\xi_{0}^{2}\left[\rho^{\prime 2}+\frac{1}{v_{\rho}^{2}} \dot{\rho}^{2}\right]\right\},
\end{aligned}
$$

where $L$ and $A$ are the wire's length and cross section, respectively, $\xi_{0}^{2}=\pi D / 8 \Delta_{0}, v_{\rho}=\sqrt{(3 \pi / 2) D \Delta_{0}}$ the amplitude velocity, $v_{\phi}=\sqrt{\pi D \Delta_{0}\left(2 A V_{c} N_{0}+1\right)}$ the phase velocity, $V_{c}$ the Fourier transform of the short range Coulomb interaction, $N_{0}$ the density of states, $D$ the electronic diffusive constant in the normal state, and the SC order parameter is parametrized as $\Delta=\Delta_{0} \rho e^{i \phi}$, with $\Delta_{0}$, the mean-field solution. For the wires in Ref. [6] $2 A V_{c} N_{0} \propto$ $N_{\perp} \gg 1$, leading to $v_{\rho} \ll v_{\phi} \propto v_{\rho} \sqrt{N_{\perp}}$. Here $N_{\perp}=$ $p_{F}^{2} A / \pi^{2}$ is the number of $1 \mathrm{D}$ channels in the wire.

This action supports QPS excitations, which are characterized by two distinct length scales: $v_{\rho} / \Delta_{0} \propto \xi \ll$ $\xi \sqrt{N_{\perp}} \propto v_{\phi} / \Delta_{0}$. For very long wires, $\xi \ll \xi \sqrt{N_{\perp}} \ll L$, in the dilute phase-slip approximation, this problem can be mapped onto the perturbative limit of the $1+1$ sineGordon model [9]. In the opposite limit of very short wires, $L<\xi \ll \xi \sqrt{N_{\perp}}$, the system resembles a JJ and can be mapped onto the $0+1$ sine-Gordon model. However the wires in Ref. [6] appear to be in the intermediate regime, $\xi \ll L \ll \xi \sqrt{N_{\perp}}$. Hence, while phase slips occur in different sections of the wire, they are indistinguishable, as each creates a phase fluctuation that spreads over distances larger than the wire itself.

Moreover, the wires in Ref. [6] have a sizable bare fugacity. Using Eq. (1), one can estimate the core action of a phase slip of duration $\tau_{0}=1 / \Delta_{0}$ by minimizing the action with respect to the diameter of a phase slip [21,22]. This yields a core action of $S_{c}=\pi / 8 \sqrt{1 / 3(1-\epsilon)} R_{Q} / R_{\xi}$. Here $\epsilon=1-T / T_{c}$ is the reduced temperature and $R_{\xi}=$ $R_{W} \xi / L$ is the normal resistance of a section of length $\xi$. With this expression, the measured values of $R_{W}$ and $L$ (Table I), and the assumption that $\xi \approx 20 \mathrm{~nm}$, the phaseslip fugacity is estimated by $\zeta=e^{-S_{c}} \approx 0.05-0.45$, for the different wires. Namely, in the critical region, $R_{W} \approx R_{Q}$, there is a dense population of phase slips which interact with one another; thus the dilute phase-slip approximation is no longer a proper description.

The flow equation for the fugacity of a phase slip anywhere in the wire is given by

$$
d \zeta / d l=\left(1-R_{Q} / R_{s}\right) \zeta,
$$

where $d l=-d \ln \Lambda$, and $\Lambda$ is the running renormalization group (RG) scale. Equation (2) treats the phase slip as occurring on an effective JJ, with $R_{s}$ being the effective shunting resistance of the entire wire. When $\zeta$ is small, $R_{s}$ will include only the effective impedance of the leads. If $\zeta$ is not very small, we will need to include in Eq. (2) additional terms of higher powers of $\zeta$, which describe interactions between QPSs. Currently, there is no complete
TABLE I. Summary of nanowire parameters, and the parameters used to fit the experimental data; $L$ is the length of the wire determined from scanning electron microscope images, $R_{W}$ is the wire's normal state resistance, taken as the resistance measured below the film transition. Fitting parameters: $R^{*}=$ $R_{\text {tot }}(T=\Delta)$ is the wire's resistance at the UV cutoff, $\Delta$ the SC order parameter, and $R_{\text {elec }}$ the impedance of the electrodes at $T<\Lambda<\Delta$.

\begin{tabular}{cccccc}
\hline \hline & \multicolumn{3}{c}{ Measured values } & \multicolumn{3}{c}{ Fitting values } \\
Curve & $L(\mathrm{~nm})$ & $R_{W}(\mathrm{k} \Omega)$ & $R^{*}(\mathrm{k} \Omega)$ & $\Delta(\mathrm{K})$ & $R_{\text {elec }}(\mathrm{k} \Omega)$ \\
\hline 1 & 177 & 5.46 & 4.2 & 2.5 & 1.2 \\
2 & 43 & 3.62 & 2.62 & 2.35 & 1.25 \\
3 & 63 & 2.78 & 2.13 & 3.07 & 0.66 \\
4 & 93 & 3.59 & 2.89 & 3.85 & 0.55 \\
5 & 187 & 4.29 & 4.5 & 6.55 & 0.31 \\
6 & 99 & 2.39 & 2.09 & 4.84 & 0.4 \\
\hline \hline
\end{tabular}

understanding of how to approach the regime of intermediate fugacity $\zeta \lesssim 1$. We suggest to account for a finite $\zeta$ by including the resistance due to other phase slips in the wire in the effective shunt resistance of the junction, $R_{s}$. This treatment was successfully tested by numerical analysis of a simpler analog of the system, an interacting pair of resistively shunted JJs [16]. Indeed, including the interaction between phase slips as a modification of the shunt, $R_{S}$, is akin to guessing the form of a complete resummation of higher-order $\zeta$ terms in Eq. (2) $[23,24]$.

The main physical intricacy is the determination of the effective shunt resistance, $R_{s}(\zeta)$, that governs the renormalization of the phase-slip fugacity [Eq. (2)]. A phase slip produces time-varying phase gradients, and hence electrical fields. These dissipate through two channels in parallel: the SC channel-which has an effective resistance due to other phase slips, $R_{\mathrm{ps}}$ - and the quasiparticles conducting channel [17], $R_{\mathrm{qp}}$. Once the disturbance reaches the leads, it also dissipates through the electrodynamical modes of the large electrodes, whose real impedance is parametrized by $R_{\text {elec }}$.

For $T \ll T_{c}$, the resistance of the quasiparticles, $R_{\mathrm{qp}}$, can be approximated by $R_{\mathrm{qp}}=\left(m / e^{2} \tau_{n} n_{\mathrm{qp}}\right)(L / A)=$ $R_{n}\left(n / n_{\mathrm{qp}}\right) \approx R_{n} \sqrt{\left(T / 2 \pi \Delta_{0}\right)} e^{\Delta_{0} / T}$. Unfortunately, we lack a microscopic model for the impedance of the electrodes, as this depends on the details of the system. However, we expect that at large scales, $T<\Lambda<\Delta$, the electrodes will act as a transmission line to the electromagnetic waves generated by the phase slip. This transmission line is characterized by a real impedance which we denote as $R_{\text {elec}}$, and use as a fitting parameter. Hence, the effective shunt resistance that affects the renormalization of the phase-slip fugacity at $T<\Lambda<\Delta$ [Eq. (2)] is

$$
R_{s}[\zeta(\Lambda)]=R_{\mathrm{elec}}+\left(1 / R_{\mathrm{ps}}[\zeta(\Lambda)]+1 / R_{\mathrm{qp}}(T)\right)^{-1} .
$$

Figure 1 shows the circuit we suggest describes the system. 


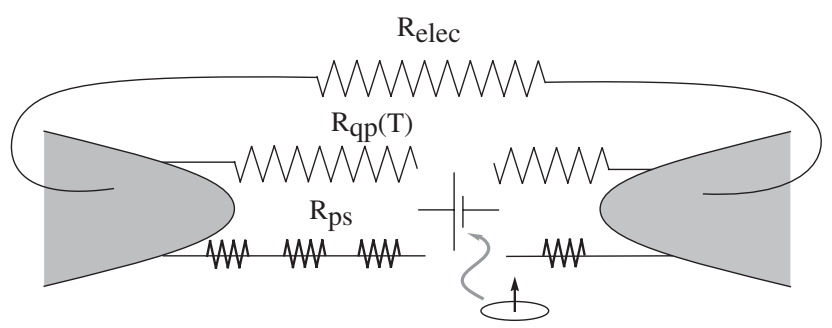

FIG. 1. The effective electrical circuit for the nanowire. A phase slip produces time-varying phase gradients, and hence electrical fields, that dissipate through the quasiparticles conducting channel, $R_{\mathrm{qp}}$, and through the SC channel, that has an effective resistance due to other phase slips, $R_{\mathrm{ps}}$. Once the disturbance reaches the edges, it also dissipates through the electrodynamical modes of the large electrodes, whose real impedance is represented by $R_{\text {elec }}$.

The resistance is measured in response to an applied dc current. At this zero frequency limit, the electrodes act as a capacitor connected in parallel to the wire. Therefore, the measured resistance is the total wire resistance, unaffected by the environment, which is cut off from the wire,

$$
R_{\mathrm{tot}}(T)=\left(1 / R_{\mathrm{ps}}[\zeta(T)]+1 / R_{\mathrm{qp}}(T)\right)^{-1} .
$$

The occurrence of a phase slip causes a resistance in the otherwise SC wire through the relation $R_{\mathrm{ps}} \propto(L / \xi) \zeta^{2}$. Using this relation and Eq. (2), we can write an RG equation for the dimensionless resistance

$$
\begin{aligned}
\frac{d \zeta^{2}}{d l} & =2 \zeta \frac{d \zeta}{d l}=2\left(1-\frac{R_{Q}}{R_{s}(\zeta)}\right) \zeta^{2} \Rightarrow \frac{d\left(R_{\mathrm{ps}} / R_{Q}\right)}{d l} \\
& =2\left(1-\frac{R_{Q}}{R_{s}\left(R_{\mathrm{ps}}\right)}\right)\left(\frac{R_{\mathrm{ps}}}{R_{Q}}\right) .
\end{aligned}
$$

If we now substitute the effective resistance in Eq. (3), depicted in Fig. 1, into the RG equation (5), we find the following self-consistent equation:

$$
\frac{d\left(\frac{R_{\mathrm{ps}}}{R_{Q}}\right)}{d l}=2\left[1-\frac{R_{Q}}{\left(R_{\mathrm{elec}}+\frac{R_{\mathrm{ps}} R_{\mathrm{qp}}(T)}{R_{\mathrm{ps}}+R_{\mathrm{qp}}(T)}\right)}\right]\left(\frac{R_{\mathrm{ps}}}{R_{Q}}\right) .
$$

Integrating Eq. (6) from the ultraviolet cutoff, $\Delta\left(T^{*}\right)=T^{*}$, to the infrared cutoff, $T$, yields $R_{\mathrm{ps}}(T)$.

The wire's dc resistance Eq. (4), calculated using Eq. (6), is plotted in Fig. 2 as a function of temperature for different $R^{*}$. Here $R^{*} \equiv R_{\text {tot }}\left(T^{*}\right)$ is the normal state resistance of the wire, at the UV cutoff $\Delta\left(T^{*}\right)=T^{*}$. We assume that the wires are thin enough such that the meanfield transition from normal to $\mathrm{SC}$ is wide, and $R^{*} \approx$ $R_{\text {tot }}\left(T_{c}^{+}\right)=R_{n}$. For simplicity, we have assumed throughout our calculation that $\Delta(T) \approx \Delta$. This assumption holds at the low temperature regime, $T \ll \Delta$, where the theory of QPSs, based on the effective action Eq. (1), is expected to be valid. The resistance of the environment is taken to be

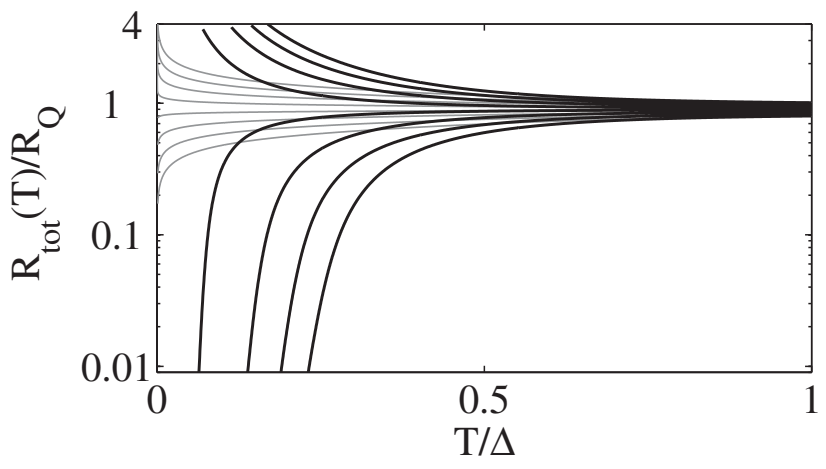

FIG. 2. Reduced resistance $R_{\text {tot }} / R_{Q}$ as a function of renormalized temperature $T / \Delta$ for different $R^{*} / R_{Q}$, ranging from 0.8 (lower plot) to 1.01 (upper plot) in increasing steps of 0.03 . The environment resistance was taken to be $R_{\text {elec }} / R_{Q}=0.1$. Gray traces indicate the total wire resistance $R_{\mathrm{tot}}=R_{\mathrm{ps}} R_{\mathrm{qp}}(T) / R_{\mathrm{ps}}+$ $R_{\mathrm{qp}}(T)$, with $R_{\mathrm{ps}}(T)=R^{*}(T / \Delta)^{2\left(R_{Q} / R_{s}-1\right)}$, and constant $R_{s}=$ $R^{*}+R_{\text {elec }}$. The insulating plots are cut off at temperature, $T_{0}$, for which $\zeta\left(T_{0}\right)=1$.

$R_{\text {elec }}=0.1 R_{Q}$. In practice, $\Delta, R^{*}$, and $R_{\text {elec }}$ can be used as fitting parameters. Moreover, $R^{*}$ can be determined independently as the resistance measured below the drop that indicates passing through $T_{c}$ of the SC films (see Ref. [6]).

Equation (6) is also applicable to a wire with $R^{*}>R_{Q}$. In this limit the fugacity increases in the renormalization process, and Eq. (2) is no longer valid for $\zeta \geqslant 1$. For wires with $R^{*}>R_{Q}$, we overestimate the phase-slip fugacity as $\zeta\left(T^{*}\right)=0.5$, and plot the reduced resistance, $R_{\text {tot }} / R_{Q}$, down to the temperature, $T_{0}$, for which $\zeta\left(T_{0}\right)=1$. The results are shown in Fig. 2. Overestimating $\zeta\left(T^{*}\right)$ gives an upper bound on $T_{0}$, where Eq. (2) is no longer applicable. Figure 2 shows that the transition between $\mathrm{SC}$ and insulating wires occurs for a critical resistance $R_{c} \approx R_{Q}$. But in contrast to the standard Josephson junction theory (gray curves in Fig. 2), the transition is much sharper (notice the $\log$ scale).

A comparison between the theoretical curves and the experimental data taken from Ref. [6] is shown in Fig. 3. The curves were calculated by fitting $R^{*}, \Delta$, and $R_{\text {elec }}$. Since the theory of QPSs is expected to be valid at $T \ll$ $\Delta$, deviations from the theoretical curves at high temperature are reasonable. In general, as $\Delta$ is proportional to $T_{c}$, increasing $\Delta$ shifts the sharp decay of the resistance to high temperatures. Both $R^{*}$ and $R_{\text {elec }}$ affect the high temperature resistance, whereas $R^{*}$ and $\Delta$ control the width of the transition.

We have made an attempt to fit the data corresponding to the insulating wires of Ref. [6]. As the insulating wires are thinner, we expect a strong suppression of $T_{c}$ [2]. Consequently, the theory of QPSs with $\zeta \lesssim 1$, is valid in a narrow range of temperatures. While we manage to fit the experimental curves in this regime of parameters, we do not present the results as our fits cover a range of $\sim 10$ data points, with three fitting parameters. 


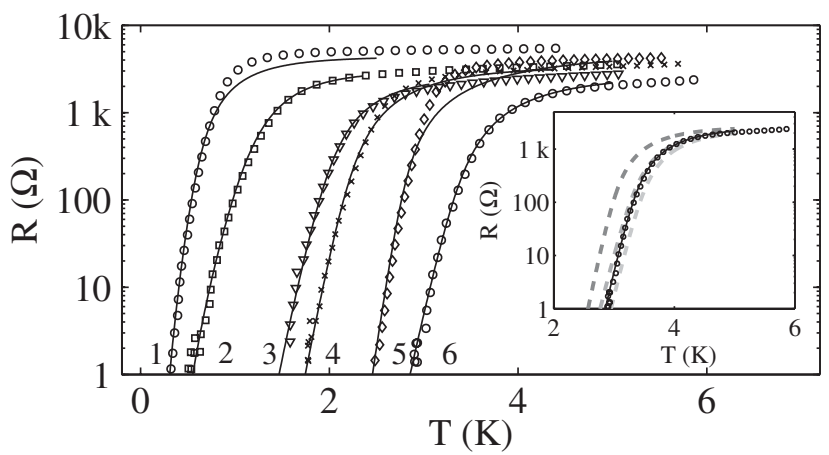

FIG. 3. Comparison between the theory (solid line) and the experimental data [6]. Details of the wires, and fitting parameters, are summarized in Table I. Note that the estimated values for $R^{*}$ are in good agreement with the measured values of the resistance of the wire at $T=\Delta$. The discrepancies between theory and experiment occur for the longer wires (curves 1 and 5). In these wires the condition $v_{\phi} / \Delta_{0} \gg L$ might not hold, and one should consider additional renormalization at higher scales. Inset: The effect of an adjustment of the fit parameters, in roughly $10 \%$ (from dark gray to light gray): $\Delta=$ $4.356 \mathrm{~K}, R_{\text {elec }}=440 \Omega$, and $R^{*}=1881 \Omega$.

In conclusion, we studied the effect of interactions between QPSs, in short SC wires, beyond the dilute phaseslip approximation. Our analysis shows that treating these interactions in a self-consistent manner produces a sharp SIT transition, with a critical resistance $R_{c} \approx R_{Q}$, in agreement with recent experiments [6]. Moreover, we have shown that adding the resistance of the Bogoliubovde Gennes quasiparticles leads to a quantitative agreement with the experimental curves. Our method should be applicable to a wider range of physical problems which involve the proliferation of topological defects with a sizable bare fugacity. In particular, it could be applied to the study of a Luttinger liquid with an extended impurity [25].

We thank E. Demler, R. A. Smith, and P. Werner. Special thanks to A. Bezryadin for making his data available to us. This study was supported by a DIP H.2.1 grant.

[1] P. Xiong, A. V. Herzog, and R. C. Dynes, Phys. Rev. Lett. 78, 927 (1997).

[2] Y. Oreg and A. M. Finkel'stein, Phys. Rev. Lett. 83, 191 (1999).

[3] F. Sharifi, A. V. Herzog, and R. C. Dynes, Phys. Rev. Lett. 71, 428 (1993).

[4] A. Bezryadin, C. N. Lau, and M. Tinkham, Nature (London) 404, 971 (2000).

[5] C. N. Lau, N. Markovic, M. Bockrath, A. Bezryadin, and M. Tinkham, Phys. Rev. Lett. 87, 217003 (2001).

[6] A. T. Bollinger, A. Rogachev, and A. Bezryadin, Europhys. Lett. 76, 505 (2006).

[7] A. Schmid, Phys. Rev. Lett. 51, 1506 (1983).
[8] S. Chakravarty, Phys. Rev. Lett. 49, 681 (1982).

[9] H.P. Büchler, V.B. Geshkenbein, and G. Blatter, Phys. Rev. Lett. 92, 067007 (2004).

[10] G. Refael, E. Demler, Y. Oreg, and D. S. Fisher, Phys. Rev. B 75, 014522 (2007).

[11] G. Refael, E. Demler, and Y. Oreg (to be published).

[12] J.S. Langer and V. Ambegaokar, Phys. Rev. 164, 498 (1967).

[13] D. E. McCumber and B. I. Halperin, Phys. Rev. B 1, 1054 (1970).

[14] The LAMH theory of thermally activated phase slips is based on the time dependent Ginzburg-Landau description of a SC wire. This description is valid at temperatures higher than the gap, and far enough from $T_{c}$, such that fluctuation corrections are small, $T^{*}<T<T_{G}$. Here $T^{*}$ is defined by $\Delta\left(T^{*}\right)=T^{*}$, with $\Delta(T)$ the temperature dependent order parameter, and $T_{G}=T_{c}(1-\mathrm{Gi})$, with $\mathrm{Gi}=\left\{\left[7 \zeta(3) / 4 \pi^{2}\right]\left[R_{\xi} / R_{Q}\right]\right\}^{2 / 3}$ the Ginzburg-Levanyuk number for the quasi-1D wires. Here $R_{\xi}=R_{W} \xi / L$ is the normal resistance of a section of length $\xi$. For the wires in Ref. [6], the applicability of LAMH is marginal as $T^{*} \approx 0.9 T_{c}$, and estimates for $T_{G}$ range between $0.85 T_{c}$ and $0.94 T_{c}$ (see Table I). This is in contrast to the well established fit of the LAMH theory to tin whisker crystals [15], where the theory is applicable for $1 \times 10^{-5}<\epsilon<$ 0.1 , and the range of fitting was $1.1 \times 10^{-4}<\epsilon<3.5 \times$ $10^{-4}$. Here $\epsilon=1-T / T_{c}$ is the reduced temperature.

[15] R. S. Newbower, M. R. Beasley, and M. Tinkham, Phys. Rev. B 5, 864 (1972).

[16] P. Werner, G. Refael, and M. Troyer, J. Stat. Mech. (2005) P12003.

[17] This could be seen by considering a time dependent Bogoliubov-de Gennes (BdG) equation, with a time dependent phase: $\Delta=|\Delta| \exp [i \phi(t)]$. By the canonical transformation $\left(u^{\prime}, v^{\prime}\right)=\exp \left(\frac{i}{2} \sigma_{z} \phi\right)(u, v)$, we produce an effective potential $\dot{\phi} / 2$ for the quasiparticles in the BdG Hamiltonian.

[18] The expansion is done in the dirty limit, $\Delta_{0} \tau \ll 1$, where $\tau$ is the scattering mean free time.

[19] D. S. Golubev and A. D. Zaikin, Phys. Rev. B 64, 014504 (2001).

[20] For a homogenous superconductor one can integrate the gap equation to obtain the first term in Eq. (1).

[21] To estimate the core action, we choose the following trial function for a phase slip: $\phi=\arctan \left(v_{\phi} \tau / x\right)$, and $\rho=\min \left[\sqrt{\left(x / x_{0}\right)^{2}+\left(\Delta_{0} \tau\right)^{2}}, 1\right]$. Identifying the part of the action [Eq. (1)] that corresponds to $\rho \neq 1$ as the core action, we minimize this expression with respect to the phase-slip diameter $x_{0}$. Keeping only leading terms in $N_{\perp}=\left(p_{F}^{2} A / \pi^{2}\right)$, one finds $S c=$ $\pi / 8 \sqrt{1 / 3(1-\epsilon)} R_{Q} / R_{\xi}$

[22] Robert A. Smith and Yuval Oreg (unpublished).

[23] Adding the next order term $a \zeta^{3}$ [24] to Eq. (2) can be thought of as modifying $R_{s}$ by $R_{s} \rightarrow R_{s}\left[1+a\left(R_{s} / R_{Q}\right) \zeta^{2}\right]$. $d \zeta / d l=\zeta-R_{Q} / R_{s} \zeta+a \zeta^{3} \approx \zeta-\zeta\left\{R_{Q} /\left[R_{s}(1+\right.\right.$ $\left.\left.\left.a R_{s} / R_{Q} \zeta^{2}\right)\right]\right\}$.

[24] S. A. Bulgadaev, Phys. Lett. A 86, 213 (1981).

[25] C. L. Kane and M. P. A. Fisher, Phys. Rev. B 46, 15233 (1992). 